\title{
MIMO Channel Analysis using DVB-T Signals
}

\author{
Holger Meuel \\ Institut fuer Nachrichtentechnik \\ TU Braunschweig \\ Braunschweig, Germany \\ mholger@yahoo.de
}

\author{
Joerg Robert \\ Institut fuer Nachrichtentechnik \\ TU Braunschweig \\ Braunschweig, Germany \\ robert@ifn.ing.tu-bs.de
}

\begin{abstract}
The second generation terrestrial transmission standard DVB-T2 introduces the application of MIMO techniques based on the well-known Alamouti scheme for improved mobile and portable reception. However, no channel models are currently available to estimate the gain of Alamouti coding in these channels. Therefore, this document presents a new approach that uses existing DVB-T networks as channel sounder. Using this approach, the complete DVB-T channel is digitized and then evaluated offline using MATLAB, which allows for accurate but time consuming algorithms. Initial measurement results already indicate the high accuracy of the developed solution. This tool can then be used in further measurement campaigns. The obtained measurement results can be incorporated in new channel models that allow for estimating the gain of multi-antenna techniques in these channels.
\end{abstract}

Keywords-Software-Radio, DVB-T, DVB-T2, Channel Modeling

\section{INTRODUCTION}

The optimization of mobile broadcasting standards is a challenging task which requires sophisticated channel models. This aspect especially gets important when new algorithms are introduced. The recently developed DVB-T2 specification [1] introduces MISO (Multiple Input, Single Output) transmission based on the Alamouti scheme [2]. The different transmitters within a Single Frequency Network (SFN) transmit differently encoded data streams at the same time and on the same frequency while a single receiving antenna is still sufficient for receiving the complete data. However, there are no sophisticated channel models for simulating the performance of such systems in the UHF band. Therefore, this paper proposes the usage of the already existing DVB-T (Digital Video Broadcasting - Terrestrial) [3] networks as channel sounder. As shown in section II, DVB-T is well-suited for this approach. Furthermore, the network structure of the new system will be most probably similar to the structure of already existing broadcasting systems. Section III then describes the evaluation of the channel estimation and the developed MATLAB software that was used for analyzing the measured data. Section IV gives initial measurement results and section $\mathrm{V}$ concludes this paper.

\section{DVB-T - AN IDEAL CHANNEL SOUNDER}

Like most state-of-the-art transmission schemes, the DVB-T standard employs OFDM (Orthogonal Frequency Division Multiplex) [4] for transmission of the data. Using
OFDM, the data is transmitted in thousands of narrow-band subcarriers. In most cases the $8 \mathrm{~K}$ FFT mode is employed for DVB-T, which has a subcarrier bandwidth of $1.1 \mathrm{kHz}$ and a total number of 6817 active subcarriers that lead to the signal bandwidth of $7.61 \mathrm{MHz}$ (in an $8 \mathrm{MHz}$ channel raster). The main advantage of this modulation scheme is the fact that each individual OFDM subcarrier is not frequency selective. Hence, if the maximum excess delay of the channel is shorter than the guard interval used, the equalization simplifies to a complex multiplication within the receiver [5].

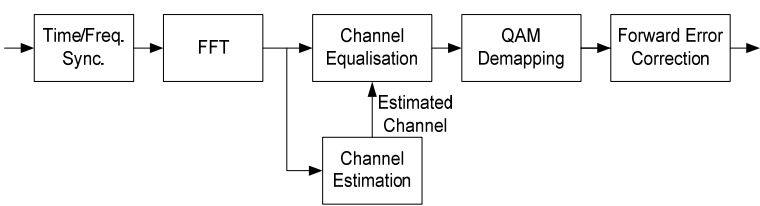

Figure 1: Simplified DVB-T receiver block diagram

Figure 1 shows a simplified DVB-T receiver block diagram. First of all, the receiver has to synchronize on the signal in time and frequency. Then it performs a Fast Fourier Transform (FFT) to obtain the individual OFDM subcarriers. Afterwards, as DVB-T uses a coherent modulation, the receiver has to equalize the subcarriers for further decoding of the signal. Therefore, it has to estimate the linear channel distortions. In case of DVB-T this aim is reached by special pilot tones that are embedded into the framing structure. These pilot tones have a specific modulation that is known to both the transmitter and the receiver. Hence, the receiver is able to estimate the channel transfer function at the pilot locations. The channel transfer function between the pilot locations is then obtained by interpolation.

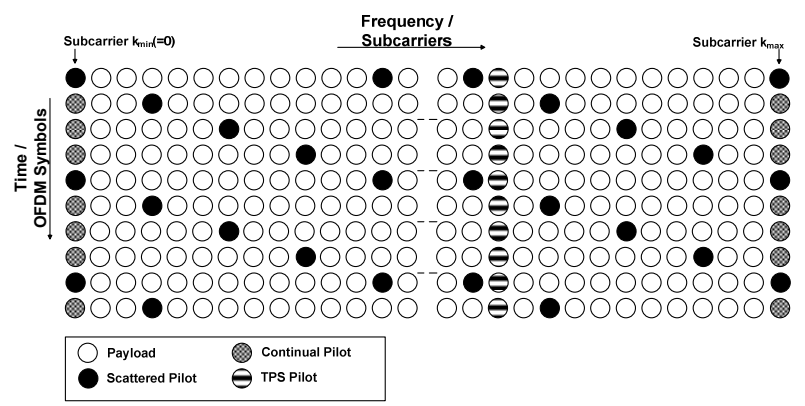

Figure 2: DVB-T framing structure 
Figure 2 shows the DVB-T framing structure in the time and the frequency direction, where each circle illustrates one OFDM subcarrier in a given OFDM symbol. The figure also shows the positions of the three different types of pilots used in DVB-T. The continual pilots are located randomly over the frequency axis, but are present in every OFDM symbol. They are especially designed for the synchronization to the signal. The TPS pilots are used for signaling purposes and are also located randomly over the frequency axis. The most important pilots for measuring the channel are the so-called scattered pilots. These pilots are transmitted on every $12^{\text {th }}$ OFDM subcarrier, while a shift of 3 OFDM subcarriers exists between the positions of the scattered pilots of two adjacent OFDM symbols. Hence, the pilot structure recurs every 4 OFDM symbols. If the channel is assumed to be quasi static, time interpolation may be used between the pilots of different OFDM symbols. This practically provides a scattered pilot on every $3^{\text {rd }}$ OFDM carrier, which allows estimating channel impulse responses of up to $300 \mu$ s within the DVB-T 8K FFT mode. Normally, these pilots are used for the equalization of the channel, but they also provide ideal means to measure the impulse response of the channel. However, further improvements are still possible and are described in the following sections.

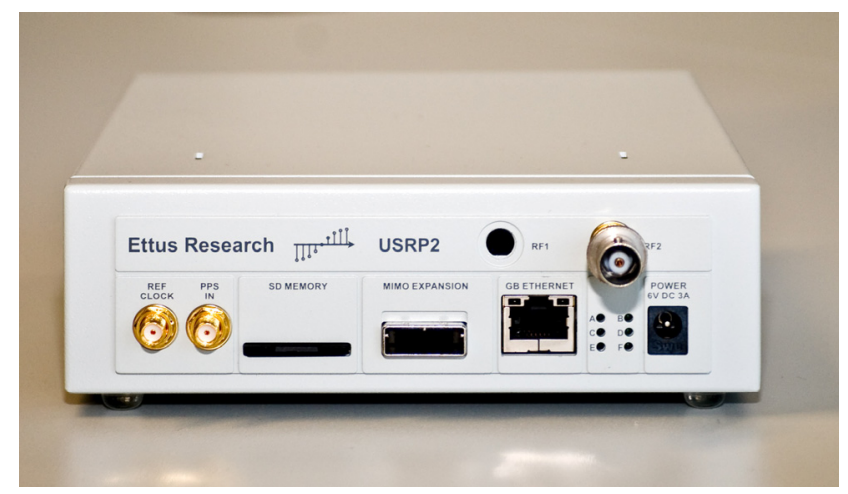

Figure 3: The Ettus Research USRP-2 software radio peripheral that has been used for measurements

\section{MEASUREMENT OF THE CHANNEL}

For the measurement of the channel a software tool was developed. A main goal of the measurement tool is the very precise estimation of the channel transfer function and the channel impulse response. For this application, a real-time evaluation of the data is not required. Hence, the channel data for the presented measurement system is sampled using a software radio peripheral, e.g. the USRP device (figure 3), a frontend available for GNU Radio [6]. Afterwards, the recorded data is evaluated offline using MATLAB, which allows for the use of accurate, but time consuming decoding algorithms that are not feasible for real-time implementations. The whole decoding process that is organized in three main steps is realized using MATLAB, which grants a high flexibility for code-improvements and additional postprocessing steps.

\section{A. Initial Channel Estimation}

The initial channel estimation is based on the scattered pilots, and is in principle similar to the channel estimation process applied in normal DVB-T receivers. First of all, the software acquires synchronization to the previously sampled channel data in time and frequency. Then it uses the scattered pilots for initially estimating the channel.

The effects of the multipath propagation on OFDM (if the maximum excess delay of the channel is shorter than the guard interval length and the channel is "slowly" varying over time) can be modeled as the multiplication of the transmitted QAM symbol $x$ in OFDM symbol $l$ at OFDM subcarrier position $k$ with the channel transfer function $H(l, k)$ :

$$
y(l, k)=x(l, k) \cdot H(l, k)+n(l, k)
$$

where the noise term $n(l, k)$ has to be added to each subcarrier. Furthermore, the channel transfer function $H$ is the Fourier transform of the channel's impulse response $h(t)$, which is naturally unknown at the beginning. However, the transmitted value $x$ is known on the pilot positions. Hence, the receiver can estimate the channel at the carrier position $p$ by:

$$
\hat{H}_{P}(l, k)=\frac{y_{P}(l, k)}{x_{P}(l, k)}-\frac{n(l, k)}{x_{P}(l, k)}
$$

while $x_{P}(l, k)$ is the modulation value of the pilot at the pilot position $p$. The channel transfer function can then be estimated by means of interpolation [4]. If in the case of DVB-T the maximum excess delay is shorter than the guard interval length and if the channel is noise-free, the estimated channel transfer function would be the ideal one. However, the additional noise term $n$ reduces the accuracy of the estimated channel transfer function. Though, this is not critical for normal receivers, as the reduced accuracy caused by the noise does not decrease the receiver's performance significantly in most cases. In contrast, the main focus of the developed system is the achievement of highest accuracy. Consequently, a modified two step approach for measuring the channel is used. Figure 4 gives an overview of this approach that allows for the reduction of the noise in the estimated transfer function.

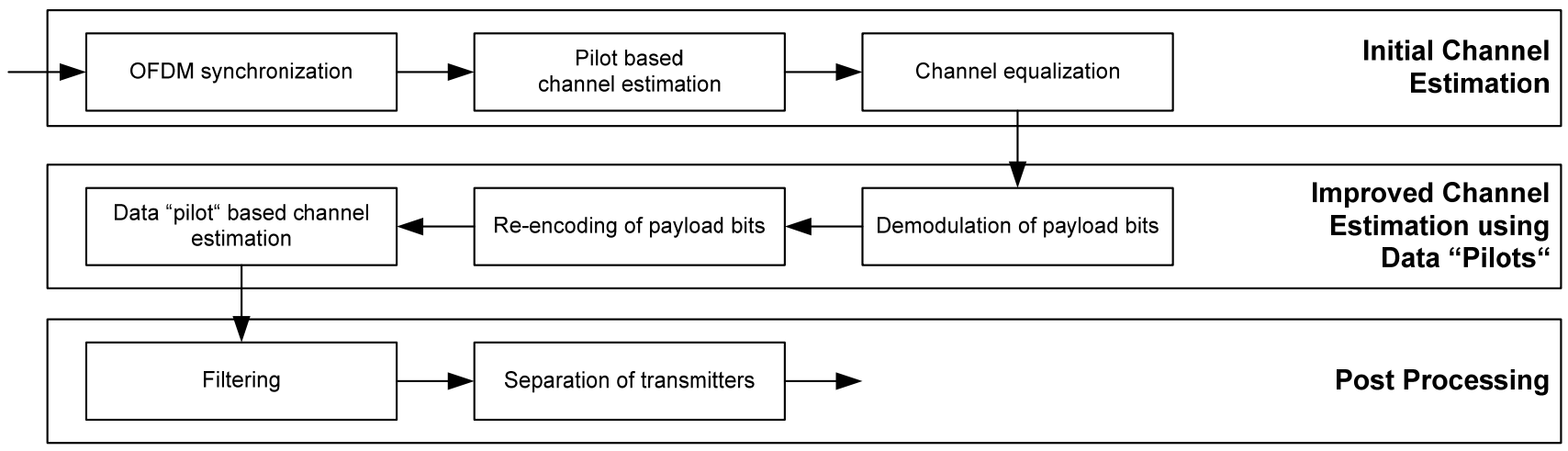

Figure 4: Principle block diagram of the implemented channel estimation chain 


\section{B. Improved Channel Estimation using Data "Pilots"}

Whilst the estimated channel is sufficient to decode the payload data correctly, the noise that is always present in real measurements reduces the quality of the measurement results significantly. Therefore, the second step uses additional processing as described in $[7,8]$ to further optimize the measured channel transfer function. After the estimation of the channel, the software is able to decode the complete data stream, which is directly encoded again afterwards. As a result, the transmitted data $x$ is known on every OFDM subcarrier, if the receiver was able to decode the data stream correctly. Hence, also the payload carriers can be used as pilot tones. Consequently, the density of the pilots is increased by a factor of 12 , which means that every OFDM subcarrier is treated as a pilot. Thus, the energy available to "pilots" is practically increased by approx. $10 \mathrm{~dB}$. Furthermore, echoes up to the length of the OFDM symbol (i.e. $896 \mu$ s) can be estimated. This maximum echo length is significantly longer than the allowed guard interval. Figure 5 and 6 show the improvement of the quality of the channel transfer function. Both figures depict the same channel transfer function, while figure 6 shows the optimized approach. It is clearly visible that the channel estimation noise is reduced drastically using the new approach.

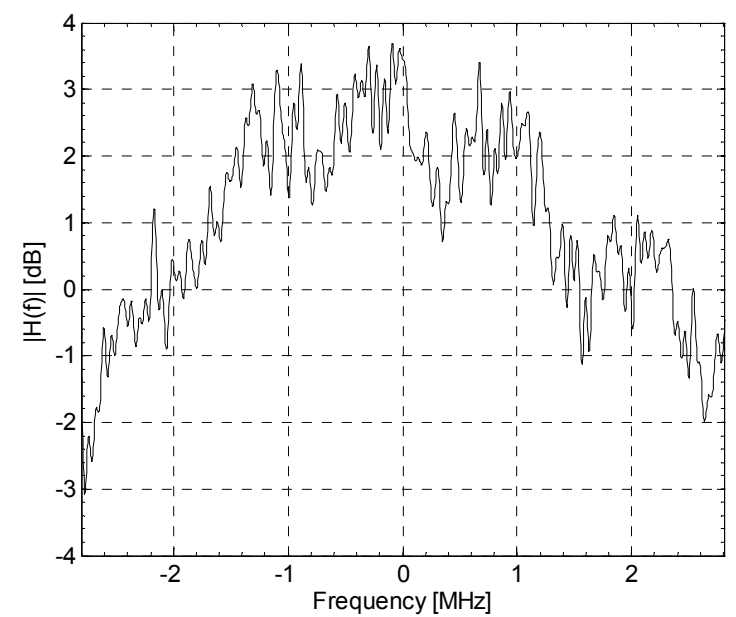

Figure 5: Pilot based channel transfer function

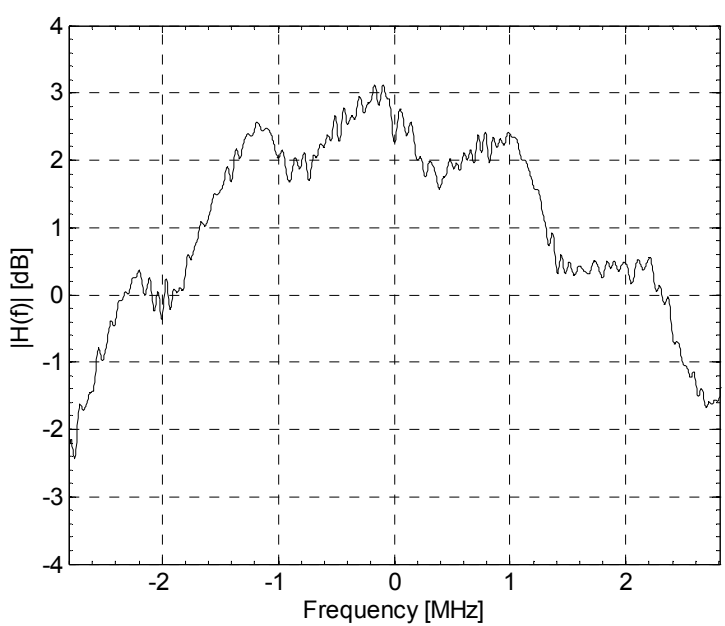

Figure 6: Improved channel transfer function using the payload data as pilots

\section{Post Processing}

The knowledge about channel statistics allows for additional improvements of the estimated channel data by means of linear filtering, i.e. the reduction of noise. First of all, a filtering can be applied inside the individual OFDM symbols. The bandwidth (or "time-width") of this filter depends on the maximum excess delay of the channel. Furthermore, also filtering in the time direction can be used, where the filter bandwidth depends on the time variance of the channel. This filtering can suppress the noise level by more than $10 \mathrm{~dB}$. The paper [9] describes this approach in detail.

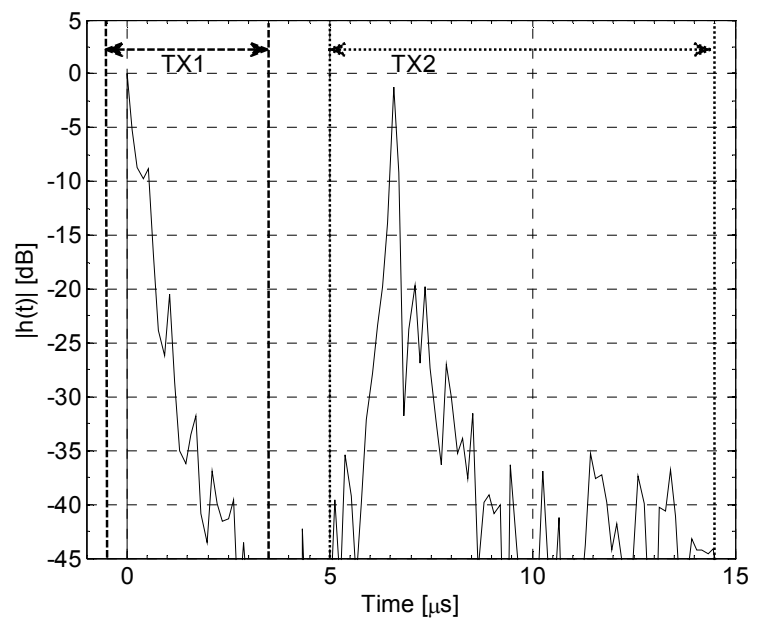

Figure 7: Separation of the signals from different transmitters within the impulse response of a Single Frequency Network by means of temporal windowing of the impulse response

One additional aspect is the separation of the different transmitters within a Single Frequency Network, which is required for modeling the effects of MIMO. As shown in figure 7, the impulse responses of the individual transmitters of a SFN are well-separated in time in most cases. Therefore, it is possible to design a filter that suppresses the signals of the other transmitters within the channel transfer function. Within the impulse response, which is the inverse Fourier transform of the channel transfer function, this can be described as windowing the signals of the individual transmitters. Thus, it is possible to measure the channel transfer function of each transmitter of the network separately. Naturally, if the impulse responses of the different transmitters overlap, this approach is not feasible.

\section{Initial MEASUREMENT Results}

Initial measurements within the DVB-T Single-FrequencyNetwork (SFN) Hannover/Braunschweig were made to prove the performance of the developed approach, where again the USRP device has been used to sample the channel. This network is built up by five transmitters in SFN operation using the DVB-T parameters $8 \mathrm{~K}$ FFT, 16-QAM with code rate $2 / 3$ and guard interval 1/4. The maximum distance of the transmitters is up to $50 \mathrm{~km}$, which is still within the range of the guard interval. 

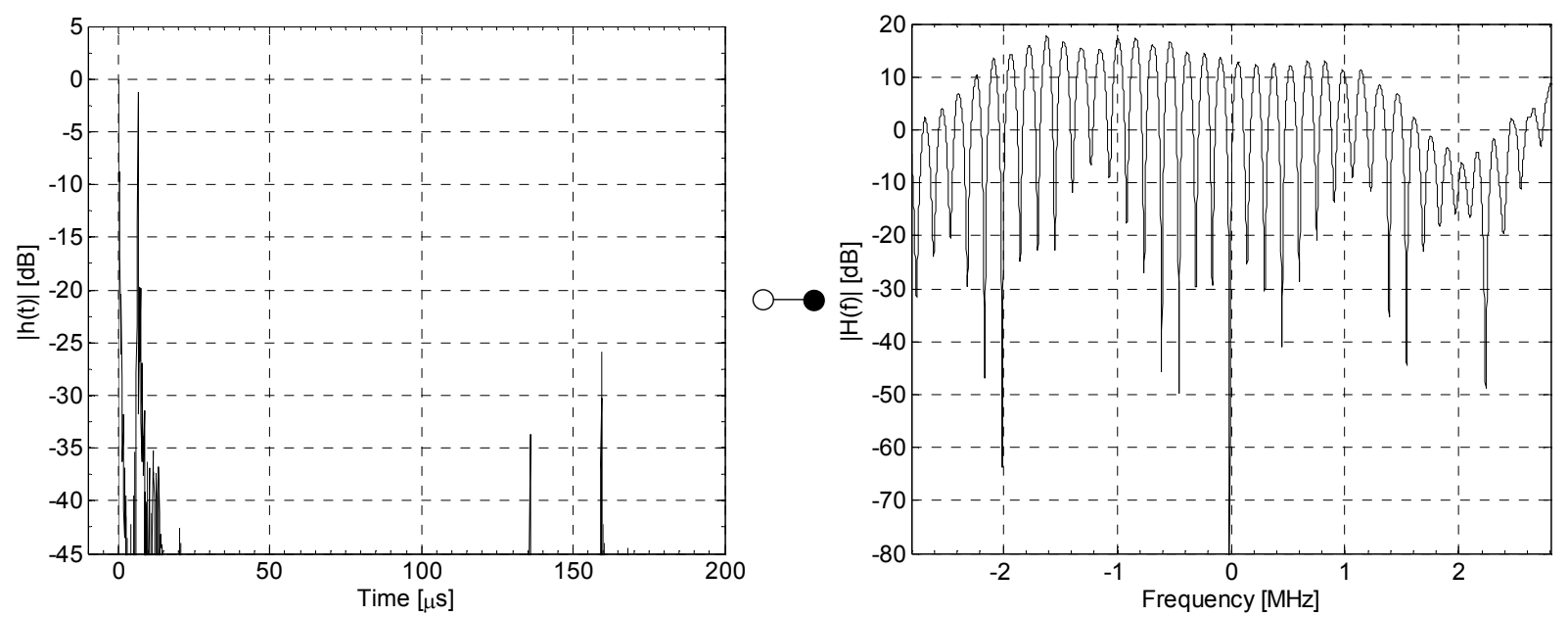

Figure 8: Measured impulse response (left) and corresponding channel transfer function in the DVB-T SFN Hannover/Braunschweig measured in Braunschweig. The two strongest signals in the impulse response are nearly at the same power level and lead to strong interference in the corresponding channel transfer function. The channel transfer function was obtained by means of the improved channel estimation process.
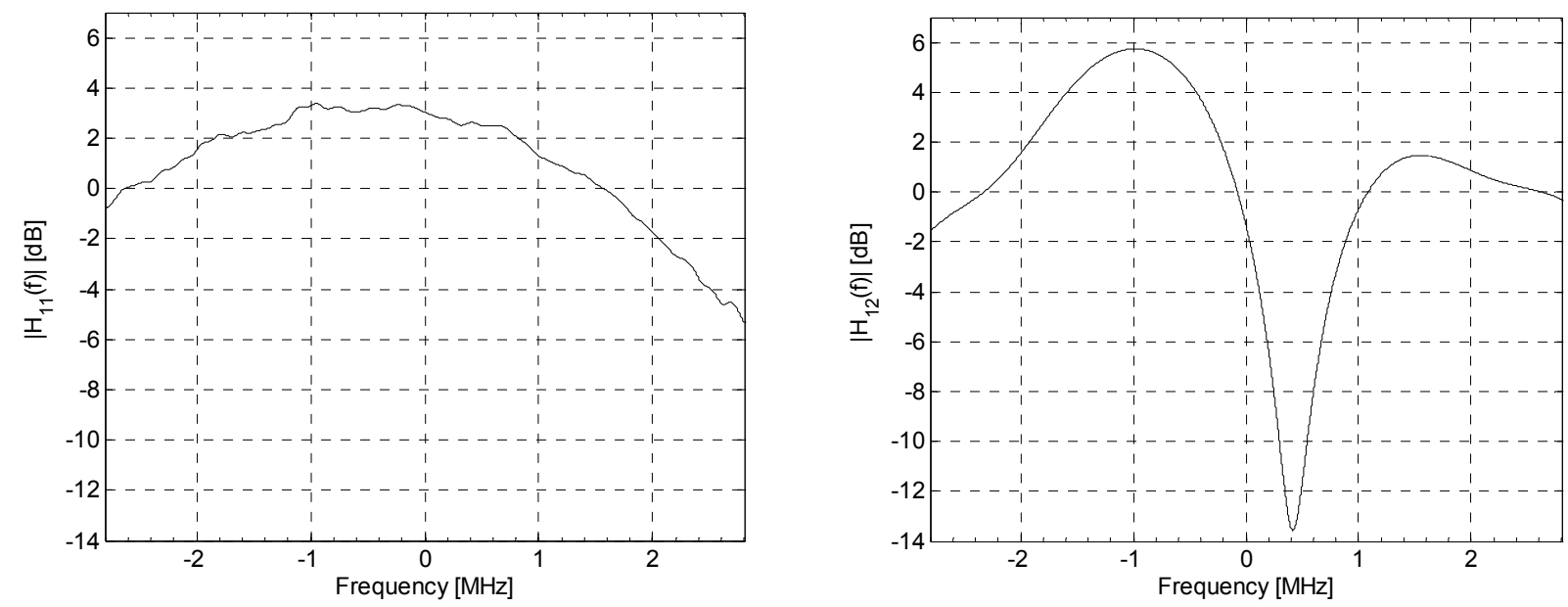

Figure 9: Separated channel transfer functions for the first and the second transmitter.
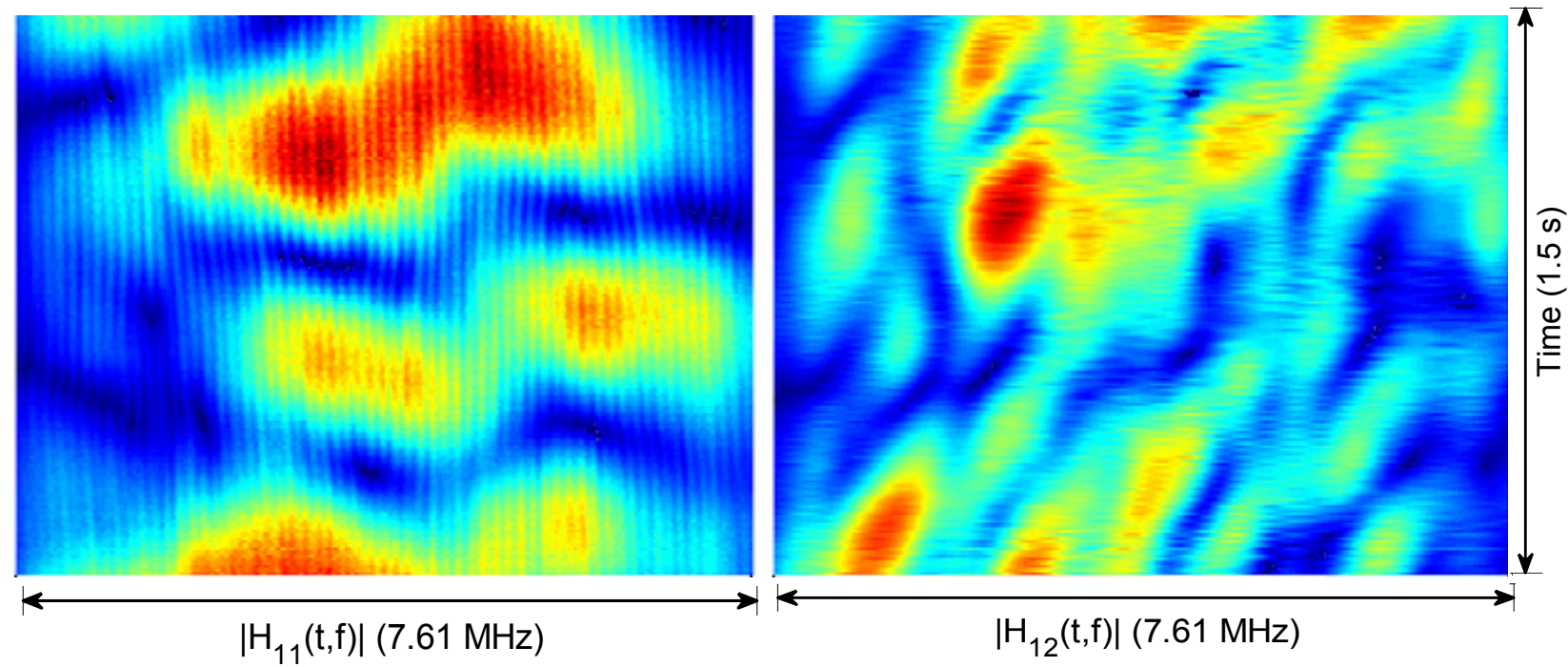

Figure 10: Simultaneously measured channel transfer functions of two DVB-T transmitters in the SFN Hannover/Braunschweig (mobile reception). Strong signal components are indicated by red, weak by blue colors. 
The first presented measurement was made indoor in a residential area of Braunschweig using the $490 \mathrm{MHz}$ channel of the local DVB-T network. A small rod-antenna on top of a TV set was used during this measurement. Figure 8 shows the impulse response obtained by using the developed software, and the corresponding channel transfer function, which is the Fourier transform of the channel impulse response. In case of the impulse response, the strongest peak has been normalized to $0 \mathrm{~dB}$, while the channel transfer function has been normalized to an average level of $0 \mathrm{~dB}$. Due to the extensive signal processing, the noise within the impulse response is more than $45 \mathrm{~dB}$ below the level of the strongest path. Hence, the impulses of 4 transmitters are clearly visible within the impulse response. The occurrence of the peaks also matches the relative distance to the different transmitters. The closest transmitter is in only $2 \mathrm{~km}$ distance, while the distance to the transmitter that caused the second peak within the impulse response is about $4 \mathrm{~km}$. The distances of the other transmitters are $43 \mathrm{~km}, 50 \mathrm{~km}$, and $52 \mathrm{~km}$, respectively. By taking the speed of light, these distances can be translated into relative delays with respect to the first signal, i.e. $7 \mu \mathrm{s}, 136 \mu \mathrm{s}, 159 \mu \mathrm{s}$, and $169 \mu \mathrm{s}$, respectively. Except the fact that the most distant transmitter is not visible, the other delays exactly fit the measured impulse response. Furthermore, the measurement also shows that the signals of the two strongest signals within the impulse response have almost identical strengths.

In case of OFDM, the data is transmitted in thousands of narrow-band subcarriers. Hence, the channel transfer function is an important means to estimate the effect of the channel. The channel transfer function is calculated out of the impulse response by its Fourier transform. Thus, echoes of equal strength lead to deep fades within the signal, which are clearly visible in figure 7 . The loss of data within these fades has to be compensated by means of the forward error correction, which leads to significantly higher signal levels that are required for error-free reception in these channels [10].

If distributed MIMO schemes are employed, each transmitter within the network may transmit its own signal. Therefore, the signal to each transmitter has to be estimated separately. However, as the impulses of the transmitters do not overlap within the SFN's impulse response in most cases, the developed software is able to separate the signals of the different transmitters within the network. The power levels of the last three main peaks of the channel impulse response are too low for accurate channel estimations in this scenario and will not be plotted in this overview. The picture on the left in figure 9 shows the channel transfer function of the main peak, which has been used for synchronization. The characteristic is nearly flat. The second transmitter is delayed by about $7 \mu \mathrm{s}$. Thus a more frequency selective channel is the result for the estimated channel transfer function $\mathrm{H}_{12}$ in figure 9 .

Figure 10 shows the application of the developed software in case of mobile reception. These measurements were made on the campus of TU Braunschweig, also using the Hannover/Braunschweig SFN at $490 \mathrm{MHz}$. Again a small rodantenna was used for the reception. One transmitter station was in line-of-sight in distance of approx. $1 \mathrm{~km}$, while the distance to the second transmitter was approx. $6 \mathrm{~km}$ without any line-ofsight. Consequently, the second transmitter suffers higher attenuation and richer scattering. Figure 10 depicts the measured channel transfer functions in this time variant channel over a time period of $1.5 \mathrm{~s}$. The mobility at pedestrian speed causes a very time and frequency selective channel that is clearly visible. These time variant characteristics of the different transmitters can then be used to model the effects of real channels in new channel models.

\section{CONCLUSIONS AND OUTLOOK}

This paper presents a tool developed at the Technische Universitaet Braunschweig for analyzing the propagation effects in DVB-T networks. The aim is reached by means of a software radio in addition to MATLAB software, which allows for the very accurate measurement of the channel. Furthermore, the software especially allows for the separate estimation of the channel transfer functions of the individual transmitters within single frequency networks, which is a requirement in order to be able to estimate the effects of MIMO transmission in these networks. Initial measurements show the high quality of the obtained results. Extensions for the application of multiple receiving antennas are currently in preparation. Furthermore, the software can be easily adapted to support other transmission standards, e.g. DVB-T2.

\section{ACKNOWLEDGEMENT}

The authors thank their colleagues at Institut fuer Nachrichtentechnik of Technische Universitaet Braunschweig, especially Prof. Reimers, for their helpful comments.

\section{REFERENCES}

[1] Digital Video Broadcasting (DVB); Frame structure channel coding and modulation for a second generation digital terrestrial television broadcasting system (DVB-T2), ETSI EN 302 755, V1.1.1, October 2008

[2] Alamouti, S.; A Simple transmit diversity technique for wireless communications; IEEE Journal on Selected Areas in Communications, Vol. 16, No. 8, October 1998

[3] Digital Video Broadcasting (DVB); Framing structure, channel coding and modulation for digital terrestrial television, ETSI EN 300744 V1.6.1, January 2009

[4] L. Hanzo, M. Münster, B.J. C.J. Choi, T. Keller, OFDM and MCCDMA for Broadband Multi-User Communications, WLANs and Broadcasting, IEEE Press, 2003

[5] Reimers, U.: DVB - The Family of International Standards for Digital Video Broadcasting (2nd Edition), Springer-Verlag Berlin Heidelberg, January 2005

[6] www.gnuradio.org (accessed $16^{\text {th }}$ April, 2010)

[7] V. Mignone, A. Morello: CD3-OFDM: A Novel Demodulation Scheme for Fixed and Mobile Receivers, IEEE Trans. Communications, Vol. 44, No. 9, September 1996

[8] L. Martínez, H. Meuel, J. Robert, et. al: Improved Robustness for Channel Estimation without Pilots for DVB-T2, IEEE International Symposium on Broadband Multimedia Systems and Broadcasting 2010, March 2010, Shanghai

[9] Baumgarten, J.; Robert, J.; Muhammad, N.; Stadelmeier, L.: Performance of Alamouti Coding in Real Measured UHF Channels, International ITH Workshop on Smart Antennas (WSA 2010), February 2010, Bremen

[10] Robert, J.: Anwendung von MISO in Gleichwellennetzen, ITG Fachtagung für Elektronische Medien, 13. Dortmunder Fernsehseminar, 17.-18.03.2009, Tagungsband S. 86-90 